\title{
Malacofauna marinha da região costeira do Canal de Săo Sebastião, SP, Brasil: Gastropoda, Bivalvia, Polyplacophora e Scaphopoda
}

\author{
Alvaro Esteves MIGOTTO ${ }^{1}$; Claudio Gonçalves TLAGO $^{1}$ \& Aimê Rachel Magenta MAGALHĀES ${ }^{2}$ \\ ${ }^{1}$ Centro de Biologia Marinha da Universidade de São Paulo \\ (Caixa Postal 83, 11600-970 São Sebastião, SP, Brasil) \\ 2 Departamento de Biologia, Universidade Federal de Santa Catarina \\ (Caixa Postal 476, 88010-970 Florianópolis, SC, Brasil)
}

Abstract: Between June 1982 and February 1983 a survey of the marine molluscs of the Channel of São Sebastião, State of São Paulo, was carried out. A total of 195 species were found: 103 gastropods, 87 bivalves, 4 chitons, and 1 scaphopod. 140 species belong to the Caribbean fauna, 22 are circumtropical or worldwide in distribution, 13 are endemic to the Brazilian coast, and 10 belong to the Patagonian fauna. Most species $(77,6 \%$ ) were found in the intertidal zone. 142 species, recorded for the region by other authors and not found in the present survey, are also listed. A reduction in the number of species was also observed comparing the present data (1982-1983) with more recent data from other authors (1987-1989). This situation is probably due to chronic pollution and other environmental impacts.

- Resumo: Um levantamento faunístico dos moluscos marinhos do Canal de São Sebastião foi realizado entre junho de 1982 e fevereiro de 1983. Um total de 195 espécies foram encontradas: 103 gastrópodes, 87 bivalves, 4 quitons e 1 escafopodo. 140 espécies pertecem a fauna caribeana, 22 são cosmopolitas ou circuntropicais, 13 são endêmicas da costa brasileira e 10 pertecem à fauna patagônica. A maioria das espécies foi encontrada na regiāo entremarés. São também listadas outras 142 espécies de moluscos registradas por outros autores para a regiāo e não reencontradas. Foi notada uma redução no número de espécies ao se comparar os dados do presente trabalho com dados mais recentes, obtidos por outros autores. Levanta-se a hipótese de que esta situação é devida a poluição crônica e outros impactos ambientais.

- Descriptors: Marine molluscs, Community composition, Intertidal environment, Check lists, Biological survey, Environmental impact, Gastropoda, Bivalvia, Polyplacophora, Scaphopoda, São Sebastiāo, Sāo Paulo, Brazil.

- Descritores: Moluscos marinhos, Composição da comunidade, Regiāo entremarés, Lista das espécies, Levantamentos biológicos, Impacto ambiental, Gastropoda, Bivalvia, Polyplacophora, Scaphopoda, São Sebastiāo: SP, Brasil.

\section{Introdução}

Os primeiros registros da malacofauna marinha do litoral norte do Estado de São Paulo são de Thering (1897), num relato de viagem científica à Ilha de São Sebastião. Duas décadas após, Luederwaldt \& Fonseca (1923) publicaram os resultados de suas excursōes à Itha de Alcatrazes, e Luederwaldt (1929 a,b), à Ilha de São Sebastião.

Morretes $(1949,1953)$, Marcus, E. $(1953,1955,1957$, 1958), Marcus, E.B.R. (1953, 1956, 1972, 1977), Marcus \& Marcus (1955, 1957, 1960), Klappenbach (1964, 1965, 
1966), Narchi (1966), Righi (1967), Ditadi (1969), Penna (1971), Montouchet (1972), Penna-Neme (1978), Penna-Neme \& Leme (1978), Duarte (1980), Morgado (1980) Penna-Neme \& Cruz-Natali (1984), Rios (1985), Domaneschi (1986), Tiago (1989), Amaral et al. (1990), Morgado et al. (1990) e Shimizu (1991) contribuíram significativamente para ampliar o conhecimento da malacofauna de São Sebastiāo.

O presente trabalho consiste em um levantamento faunístico dos moluscos marinhos da região de São Sebastiâo. Visando apresentar uma lista abrangente, os registros dos autores citados acima foram também incluídos, nos casos das espécies não reencontradas.

A regiāo estudada e o Canal de São Sebastião como um todo sofrem interferência ambiental crônica com os pequenos, mas constantes, vazamentos de petroleo, a descarga de esgotos domésticos sem tratamento e o afluxo de material terrígeno particulado de origem antrópica (Schaeffer-Novelli, 1990). Entre 1978 e 1989 foram registrados 76 acidentes com petróleo em São Sebastiāo, relacionados às atividades do Terminal Marítimo Almirante Barroso - TEBAR, da PETROBRÁS, que liberaram para 0 ambiente marinho um total estimado de $11.000 \mathrm{~m}^{3}$ (fonte CETESB-GOE/DAEM, 1989). A Baía do Araçá e a Praia do Cabelo Gordo de Dentro foram muito afetadas por serem ambas abrigadas, de sedimento fino (cf. Amaral et al., 1990) e, portanto, muitos sensíveis à poluição por 6́leo (API, 1985; Schaeffer-Novelli, 1990).

Em 1988, parte da Baía do Araçá foi aterrada para a futura ampliaçāo do Porto de Sāo Sebastiāo. Em 1989, a dragagem de um canal, para a instalação do emissário submarino de esgostos domésticos da cidade, causou profundo impacto no restante da baía, formando várias ilhotas de areia, alterando o perfil das praias e as áreas de sedimentação, assoreando costōes rochosos, e soterrando os pneumatoforos de Laguncularia racemosa e Avicennia schaueriana.

Tendo em vista os inúmeros impactos ambientais que o Canal de São Sebastiāo vem sofrendo, um melhor conhecimento faunístico e florístico é necessário para que se possa avaliar a extensão destes impactos. Sob este enfoque, os dados deste trabalho foram comparados, quando possfvel, aos de Amaral et al.(1990) e de Shimizu (1991), obtidos cerca de cinco anos após o período em que coletamos nossas amostras, visando verificar se houve alteração no número de espécies de moluscos da região entremarés de algumas das praias estudadas.

\section{Material e métodos}

As coletas foram qualitativas e realizadas entre junho de 1982 e fevereiro de 1983. Foram considerados somente os espécimes vivos.
A amostragem na regiāo entremarés foi feita durante marés baixas de sizígia. Em substrato consolidado foram utilizadas pinças, ponteiras de aço e martelos. Nas praias arenosas as coletas foram feitas através de escavaçōes com auxilio de pá, em locais onde haviam vestígios indicativos da presença de moluscos, como rastros e orifícios de galerias. Além disso, cavamos em diferentes locais das praias, até a profundidade de $30 \mathrm{~cm}$ no substrato, e triamos o sedimento coletado em peneira com malha de $1 \mathrm{~mm}$, selecionando os moluscos nele presentes.

No infralitoral foi empregado mergulho livre e autônomo. No substrato rochoso, foi usado o mesmo procedimento da região entremarés. O substrato nāoconsolidado foi amostrado de maneira não quantitativa, em pontos situados a $5 \mathrm{e} \mathrm{a} 10 \mathrm{~m}$ de profundidade; as coletas foram realizadas, em mergulho autônomo, com o auxílio de pá, até a profundidade de $30 \mathrm{~cm}$ no sedimento. $O$ material coletado foi transferido para sacos de náilon e, posteriormente, triado em peneira com malha de $1 \mathrm{~mm}$.

Além dos moluscos coletados diretamente, seixos, madeira, algas e corais, dentre outros materiais e organismos foram levados ao laboratório e examinados na busca de espécimes.

Os animais foram anestesiados em solução aquosa de cloreto de magnésio a 7,5\% ou de propilenofenoxetol, fixados em formol salino $4 \%$ e conservados em álcool $80 \%$.

A identificação preliminar das espécies foi baseada em Abbott (1974) e Rios (1985)e, posteriormente, confirmada por comparaçōes com material da coleçāo do Museu de Zoologia da Universidade de São Paulo (MZUSP) e com o auxilio de especialistas.

\section{A regiấo de estudo}

O Canal de São Sebastiāo (São Sebastião, SP), com mais de $20 \mathrm{~km}$ de extensão, estende-se entre o continente e a Illha de São Sebastiāo no eixo NE/SW; sua largura é de aproximadamente $7 \mathrm{~km}$ na entrada sul, $6 \mathrm{~km}$ na entrada norte e $2 \mathrm{~km}$ na regiāo central (Furtado, 1978). Está situado numa zona de transição, denominada Província Zoogeográfica Paulista, com limite norte entre os Estados do Espírito Santo e do Rio de Janeiro, e sul, entre o Rio Grande do Sul e o Uruguai. Esta província abriga representantes da fauna caribeana e da fauna patagônica, além de espécies circuntropicais e endêmicas (Palacio, 1982).

A regiāo de estudo abrange a porção sul do Canal de São Sebastião (Fig. 1), da Ponta do Guaecá (2350'S, $\left.45^{\circ} 27^{\prime} \mathrm{W}\right)$ à Baía do Araçá $\left(23^{\circ} 48^{\prime} \mathrm{S}, 45^{\circ} 24^{\prime} \mathrm{W}\right)$, incluindo a região entremarés e o infralitoral de nove costōes rochosos, de sete praias arenosas, de uma baía arenolodosa (Baía do Araçá) e de uma laje rochosa, parcialmente emersa (Laje dos Moleques ou Farol dos Moleques). 


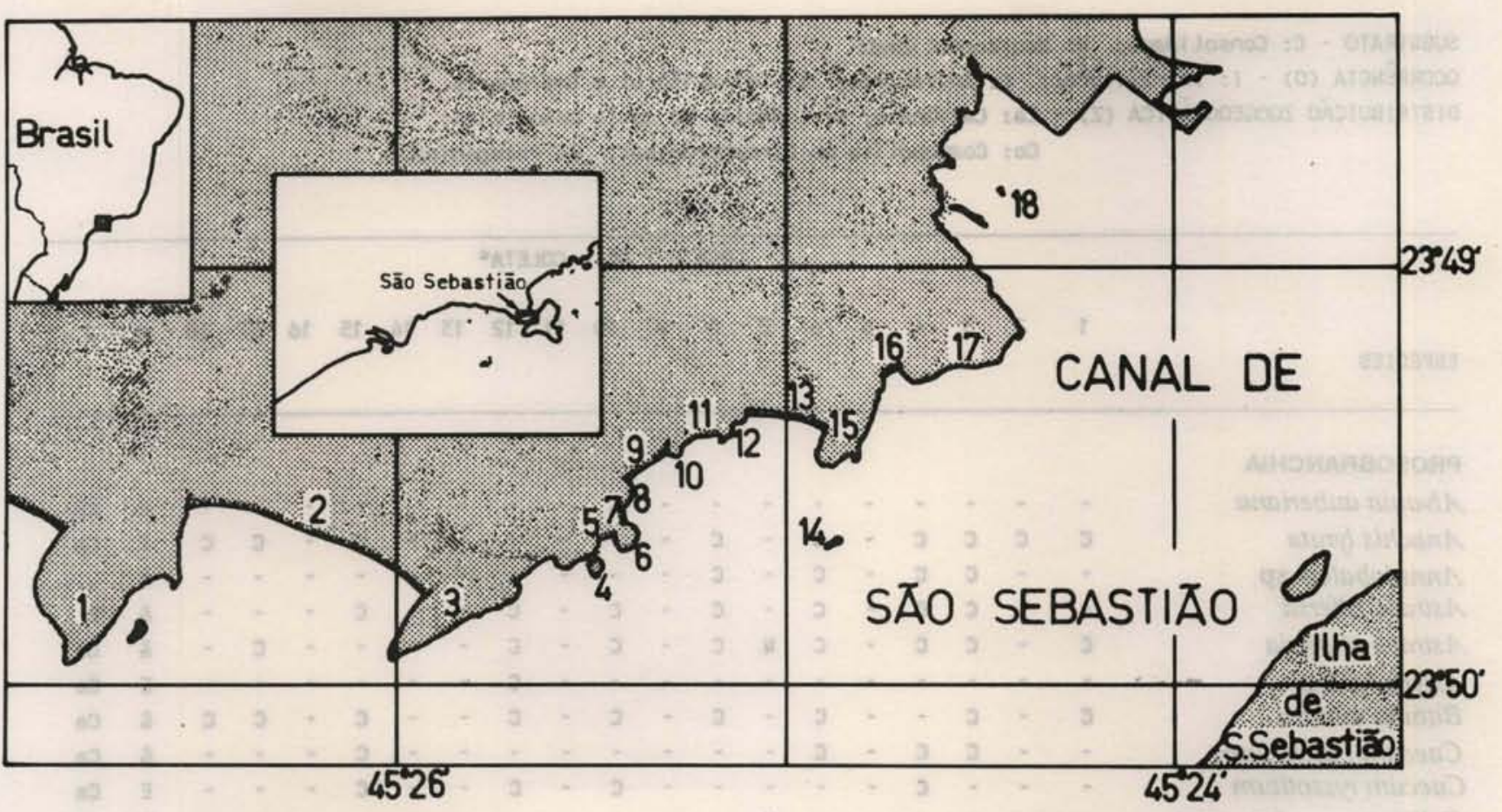

Fig. 1. Locais de coleta.

1. Ponta do Guaecá; 2. Prala de Barequeçaba; 3. Costão Praia de Barequeçaba/Ponta do Baleelro; 4. Ponta do Baleeiro; 5. Praia do Segredo; 6. Ponta do Jarobá; 7. Praia do Cabelo Gordo de Dentro; 8. Costão Praia do Cabelo Gordo/Prala das Pitangueiras; 9. Prala das Pltangueiras; 10. Costăo Praila das Pitangueiras/Praia do Zimbro; 11. Prala do Zlmbro; 12. Costão Praia do Zimbro/Praia Grande; 13. Praia Grande; 14. Laje dos Moleques; 15. Costão Prala Grande/Prala Preta; 16. Praia Preta; 17. Costão Praia Preta/Baia do Araçá. 18. Bala do Araçá.

A temperatura da água superficial do canal, próximo ao laboratório do CEBIMar, variou, entre março de 1979 e março de 1991 , de 16,5 a $31,0^{\circ} \mathrm{C}$ (média de $23,7^{\circ} \mathrm{C}$ ) e a salinidade, entre 29 e 36 (média de 33,9 ); a temperatura do ar variou de 9 a $39^{\circ} \mathrm{C}$ (média de $24,5^{\circ} \mathrm{C}$ ) (CEBIMar, 1991).

\section{Resultados}

Foram coletadas 195 espécies de moluscos: 103 gastrópodes, 87 bivalves, 4 poliplacóforos, e 1 escafópodo (Tabs 1, 2 e 3). Destes, 185 foram identificados a nível de espécie e 10, a nível de gênero.

Do total identificado a nível específico, 140 espécies (70 gastrópodes, 67 bivalves, 2 poliplacóforos e 1 escafópodo) pertencem à fauna caribeana, 22 (11 gastrópodes e 11 bivalves) são cosmopolitas ou circuntropicais, 13 ( 8 gastrópodes, 4 bivalves e 1 poliplacóforo) são endêmicas da costa brasileira e 10 (5 gastrópodes, 4 bivalves e 1 poliplacóforo) pertencem à fauna patagônica.

Os moluscos mais comuns (encontrados em pelo menos oito locais de coleta) foram os gastrópodes Anachis lyrata, Astraea phoebia, Bittium varium, Cerithium atratum, C. eburneum, Collisella subrugosa, Costoanachis catenata, C. sertulariarum, Crepidula plana, Diodora patagonica, Fissurella clenchi, Leucozonia nassa, Littorina flava, Mitrella argus, Monula nodulosa, Nodilittorina lineolata, Olivella minuta, Rissoina catesbyana, Siphonaria hispida, Tegula viridula, Thais haemastoma e Tricolia affinis, os bivalves Arca imbricata, Arcopsis adamsi, Barbatia candida, Brachidontes solisianus, Chama congregata, Corbula sp, Crassostrea mizophorae, Cyclinella tenuis, Hiatella artica, Modiolus carvalhoi, Perna perna, Pinctata imbricata e Strigilla carnaria, e o poliplacóforo Ischinochiton striolatus.

A maioria das espécies $(77,6 \%)$ foi encontrada na regiâo entremarés. Estavam presentes exclusivamente no 
Tabela 1. Gastrópodes do Canal de São Sebastião, por local de coleta

SUBSTRATO - C: Consol idado; N: Não-consol idado OCORRÊNCIA (O) - I: Infral itoral; E: Entremarés; \&: Infral itoral e Entremarés DISTRIBUIÇĂo ZOOGEOGRÁFICA (Z) - Ca: Caribeana; B: Endémica da costa brasileira; Co: Cosmopolita ou Circuntropical; P: Patagônica.

ESPECIES

\section{LOCAIS DE COLETA*}

\begin{tabular}{|c|c|c|c|c|c|c|c|c|c|c|c|c|c|c|c|c|c|c|c|c|}
\hline Alvania auberiana & - & - & - & - & & - & - & - & - & C & - & - & - & - & - & - & - & - & E & $\mathrm{Ca}$ \\
\hline Anachis lyrata & C & C & C & C & & C & - & C & & - & - & - & - & - & c & - & C & c & E & $\mathrm{Ca}$ \\
\hline Annulobalcis sp & - & - & C & C & & C & - & c & & - & - & - & - & - & - & - & - & - & I & ... \\
\hline Astraea olfersï & - & - & c & c & 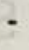 & c & - & c & - & c & - & c & - & - & c & - & - & - & $\&$ & B \\
\hline Astraea phoebia & C & - & C & C & - & C & N & C & - & C & - & C & - & - & - & - & C & - & \& & $\mathrm{Ca}$ \\
\hline Astyris lunata & & - & - & - & & - & - & - & & - & - & c & - & - & - & - & - & - & E & $\mathrm{Ca}$ \\
\hline Bittium varium & C & - & C & - & - & C & - & C & - & C & $\cdot$ & c & - & - & C & - & C & C & \& & $\mathrm{Ca}$ \\
\hline Caecum pulchellum & - & - & c & c & - & C & - & $\cdot$ & - & - & - & - & - & $\cdot$ & c & - & - & - & $\&$ & $\mathrm{Ca}$ \\
\hline Caecum ryssotitum & $\cdot$ & - & - & C & - & - & - & - & - & C & - & c & - & - & c & - & - & - & E & $\mathrm{Ca}$ \\
\hline Calliostoma adspersum & - & - & - & - & - & - & N & - & & $\cdot$ & - & $\cdot$ & - & - & - & - & - & - & \& & $\mathrm{Ca}$ \\
\hline Calliostoma euglyptum & C & - & C & - & - & c & - & - & & C & $\cdot$ & - & - & - & $\cdot$ & - & - & - & $\&$ & $\mathrm{Ca}$ \\
\hline Calliostoma jujubinum & - & - & C & C & - & C & - & - & 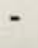 & c & - & - & - & - & $\cdot$ & 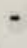 & - & - & $\&$ & $\mathrm{Ca}$ \\
\hline Calliostoma pulchrum & - & - & C & - & - & - & - & - & & - & - & - & - & - & - & - & $\cdot$ & $\cdot$ & \& & $\mathrm{Ca}$ \\
\hline Calyptraea centralis & - & $\cdot$ & - & - & - & - & N & - & & - & - & - & - & - & - & & - & - & 1 & $\mathrm{Ca}$ \\
\hline Cerithiopsis emersoni & c & - & - & - & N & - & - & - & & - & - & $\cdot$ & - & - & - & - & C & - & 1 & $\mathrm{Ca}$ \\
\hline Cerithiopsis greenii & C & - & - & $\cdot$ & - & - & - & c & - & C & - & $\cdot$ & - & - & $\cdot$ & & - & - & E & $\mathrm{Ca}$ \\
\hline Cerithium atratum & - & - & C & C & N & C & N & - & 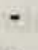 & - & - & - & - & C & c & 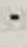 & - & C & $\&$ & $\mathrm{Ca}$ \\
\hline Cerithium ebumeum & - & $\mathbf{N}$ & C & - & $N$ & C & - & C & - & - & - & C & - & - & C & & - & - & \& & $\mathrm{Ca}$ \\
\hline Clathrodrillia sp & - & - & C & $\cdot$ & - & - & - & - & & - & - & $\cdot$ & - & $\cdot$ & - & 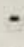 & - & - & $\mathbf{E}$ & ... \\
\hline Collisella subrugosa & C & - & C & C & - & C & - & c & . & C & - & C & - & - & C & 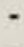 & C & C & $\mathbf{E}$ & B \\
\hline Columbella mercatoria & - & $\cdot$ & - & $\cdot$ & - & - & - & - & 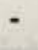 & $\cdot$ & - & - & $\cdot$ & $\cdot$ & C & - & C & C & E & $\mathrm{Ca}$ \\
\hline Costoanachis catenata & c & - & - & C & - & C & - & C & 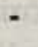 & C & - & C & - & - & C & - & - & C & \& & $\mathrm{Ca}$ \\
\hline Costoanachis sertularianum & c & c & c & C & - & C & N & c & - & c & - & c & - & - & c & - & C & C & \& & $\mathbf{P}$ \\
\hline Crepidula aculeata & c & - & - & C & - & C & - & C & - & - & - & - & - & C & - & - & C & - & $\mathbf{E}$ & Co \\
\hline Crepidula plana & C & - & - & - & C & - & C & - & C & - & c & - & C & - & c & - & C & C & $\mathbf{E}$ & Ca \\
\hline Cryoturris serga & - & $\cdot$ & - & - & - & - & - & - & - & - & N & $\cdot$ & - & $\dot{-}$ & - & & - & $=$ & 1 & Ca \\
\hline Cymatium parthenopeum & - & - & - & C & N & c & $\cdot$ & - & - & $\cdot$ & $\cdot$ & C & $\cdot$ & c & c & 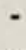 & - & C & I & co \\
\hline Cyphoma signatum & - & - & - & C & $\cdot$ & C & - & - & $\cdot$ & - & - & - & - & c & $\cdot$ & 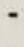 & - & 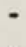 & 1 & Ca \\
\hline Cypraea zebra & - & - & - & C & - & C & - & - & - & - & - & - & - & $\cdot$ & $\cdot$ & - & - & - & $\&$ & $\mathrm{Ca}$ \\
\hline Diodora jaumei & - & - & $\cdot$ & - & - & - & - & - & - & - & - & - & - & - & C & - & - & - & E & $\mathrm{Ca}$ \\
\hline Diodora patagonica & c & - & - & c & - & C & - & C & - & C & $\cdot$ & c & - & - & c & $\theta$ & C & C & E & $\mathbf{P}$ \\
\hline Engina turbinella & c & $\cdot$ & C & - & $\cdot$ & C & - & C & - & C & - & c & - & - & c & - & - & - & \& & Ca \\
\hline Epitonium albidum & - & - & - & - & - & - & - & - & - & - & - & C & - & - & $\cdot$ & - & - & - & E & Ca \\
\hline Epitonium angulatum & - & - & C & - & - & - & - & - & - & c & - & $\cdot$ & - & - & $\cdot$ & - & - & - & E & Ca \\
\hline Epitonium novangliae & - & - & N & - & - & - & - & - & - & - & - & - & - & - & - & 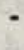 & - & - & 1 & $\mathrm{Ca}$ \\
\hline Eulima sp & - & - & - & - & - & N & - & - & - & - & - & - & - & - & - & - & - & - & I & $\cdots$ \\
\hline Favartia cellulosa & - & - & $\dot{-}$ & c & - & C & - & - & - & - & - & - & & C & - & - & - & - & 1 & Ca \\
\hline $\begin{array}{l}\text { Finella dubia } \\
\text { Fissurella clenchi }\end{array}$ & - & - & N & - & $\mathbf{N}$ & - & - & c & N & - & - & - & & - & - & 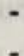 & C & $\mathbf{N}$ & \& & Ca \\
\hline Fusinus marmoratus & - & - & - & - & 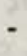 & C & 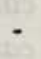 & - & - & - & - & - & & - & - & 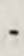 & 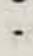 & 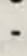 & I & co \\
\hline Hastula cinerea & - & N & - & - & - & - & - & - & & & & & & - & - & & & - & E & $\mathrm{Ca}$ \\
\hline
\end{tabular}


Tabela 1. Continuação

LOCAIS DE COLETA*

ESPECIES

$\begin{array}{llllllllllllllllllll}1 & 2 & 3 & 4 & 5 & 6 & 7 & 8 & 9 & 10 & 11 & 12 & 13 & 14 & 15 & 16 & 17 & 18 & 0 & 2\end{array}$

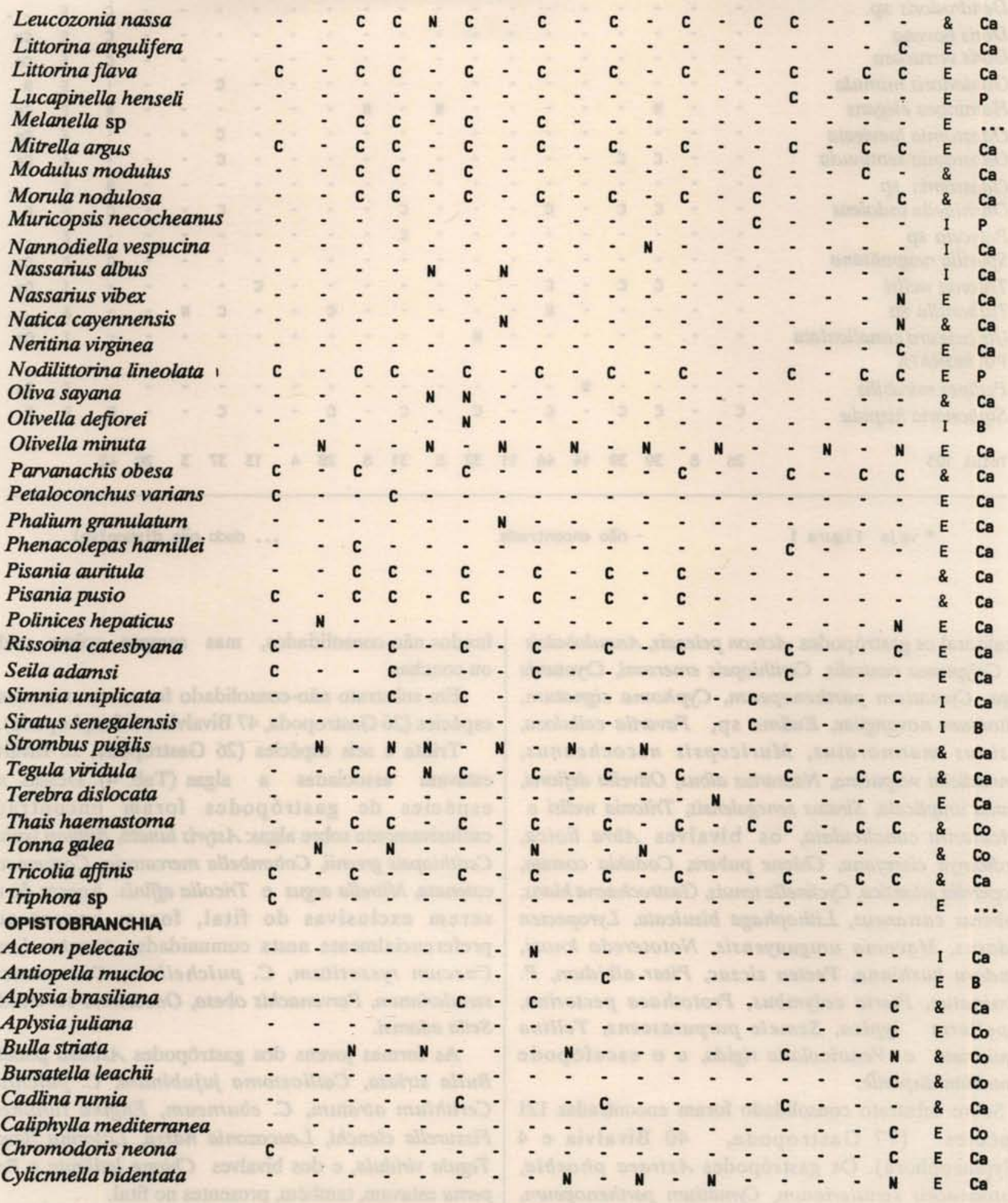


Tabela 1. Continuação

LOCAIS DE COLETA*

ESPECIES

$\begin{array}{llllllllllllllllllll}1 & 2 & 3 & 4 & 5 & 6 & 7 & 8 & 9 & 10 & 11 & 12 & 13 & 14 & 15 & 16 & 17 & 18 & 0 & 2\end{array}$

Dendrodoris sp

Doris bovena

Doris verrucosa

Goniodoris mimula

Haminoea elegans

Odostomia laevigata

Odostomia seminuda

Odostomia sp

Onchidella indolens

Polycera sp

Spurilla neapolitana

Tritonia wellsi

Turbonilla sp

Utriculastra canaliculata

PULMONATA

Pedipes mirabilis

Siphonaria hispida

Total 103

$$
\begin{aligned}
& \text { - }
\end{aligned}
$$

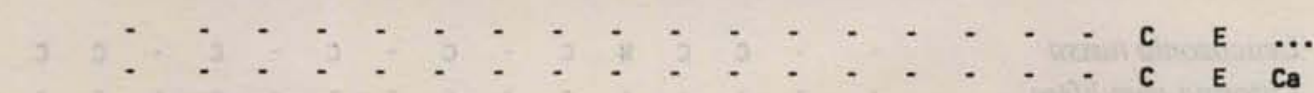

$$
\begin{aligned}
& -C_{-}-C_{-}-C_{-}-C_{-}-C_{\mathrm{C}} \mathrm{CO}
\end{aligned}
$$

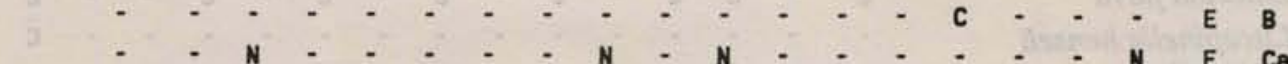

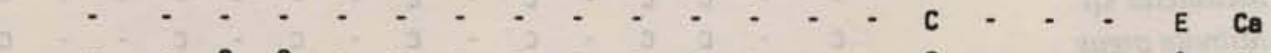

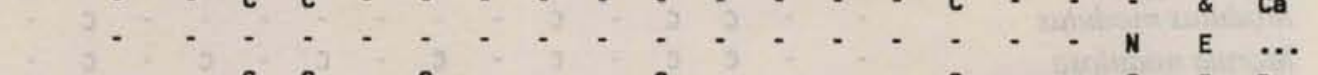

$$
\begin{aligned}
& \text { C E B } \\
& \text { - E } \ldots \\
& \text { C E Co } \\
& \text { - I Ca } \\
& \text { - } \& \text { \& } \\
& \text { - I Ca } \\
& \text { E Ca } \\
& C-C \mathrm{C}-\mathrm{C}-\mathrm{C}-\mathrm{C}-\mathrm{C}-\mathrm{C}-\mathrm{C}_{\mathrm{B}} \\
& \begin{array}{llllllllllllllllll}
26 & 8 & 39 & 39 & 14 & 46 & 11 & 32 & 8 & 31 & 8 & 28 & 4 & 13 & 37 & 3 & 20 & 42
\end{array}
\end{aligned}
$$

* veja Figura 1

- não encontrada
... dado não disponf́vel infralitoral os gastropodes Acteon pelecais, Annulobalcis sp, Calyptraea centralis, Cerithiopsis emersoni, Cryoturris serga, Cymatium parthenopeum, Cyphoma signatum, Epitonium novangliae, Eulima sp, Favartia cellulosa, Fusinus marmoratus, Muricopsis necocheanus, Nannodiella vespucina, Nassarius albus, Olivella defiorei, Simnia uniplicata, Siratus senegalensis, Tritonia wellsi e Utriculastra canaliculata, os bivalves Abra lioica, Cardiomya clereyana, Chione pubera, Codakia costata, Cooperella atlantica, Cyclinella tenuis, Gastrochaena hians, Lioberus castaneus, Lithophaga bisulcata, Lyropecten nodosus, Macoma uruguayensis, Nototeredo knoxi, Pandora bushiana, Pecten ziczac, Pitar albidum, P. fulminatus, Pteria colymbus, Protothaca pectorina, Rupellaria typica, Semele purpurascens, Tellina aequistriata e Ventricolaria rigida, e o escafópode Dentalium disparile.

Sobre substrato consolidado foram encontradas 121 espécies (77 Gastropoda, 40 Bivalvia e 4 Polyplacophora). Os gastrópodes Astraea phoebia, Costoanachis sertularianum, Cymatium parthenopeum, Leucozonia nassa e Tegula viridula, típicos de substratos rochosos, foram encontrados também em fundos não-consolidados, mas sempre sobre pedras ou conchas.

Em substrato nāo-consolidado foram encontradas 74 espécies (26 Gastropoda, 47 Bivalvia e 1 Scaphopoda).

Trinta e seis espécies (26 Gastropoda, 10 Bivalvia) estavam associadas a algas (Tab. 4). Dessas, sete espécies de gastrópodes foram encontradas exclusivamente sobre algas: Astyris lunata, Bittium varium, Cerithiopsis greenii, Columbella mercatoria, Costoanachis catenata, Mitrella argus e Tricolia affinis. Apesar de näo serem exclusivas do fital, foram encontradas preferencialmente nesta comunidade: Anachis lyrata, Caecum ryssotitum, C. pulchellum, Costoanachis sertularianum, Parvanachis obesa, Odostomia seminuda e Seila adamsi.

As formas jovens dos gastrópodes Astraea phoebia, Bulla striata, Calliostoma jujubinum, C. pulchrum, Cerithium atratum, C. ebumeum, Engina turbinella, Fissurella clenchi, Leucozonia nassa, Littorina flava e Tegula viridula, e dos bivalves Chione latilirata e Perna perna estavam, também, presentes no fital.

Catorze espécies estavam associadas a invertebrados (Tab. 5), sete das quais de modo exclusivo. Jovens de 
Tabela 2. Bivalves do Canal de São Sebastião, por local de coleta

SUBSTRATO - C: Consol idado; N: Nào-consol idado

OCORRÊNCIA ( 0 ) - I: Infral itoral; E: Entremarés; \&: Infral itoral e Entremarés

DISTRIBUIÇÃO ZOOGEOGRÁFICA (Z) - Ca: Caribeana; B: Endêmica de costa brasileira;

Co: Cosmopolita ou Circuntropical; P: Patagônica.

ESPECIES

LOCAIS DE COLETA*

$\begin{array}{llllllllllllllllllll}1 & 2 & 3 & 4 & 5 & 6 & 7 & 8 & 9 & 10 & 11 & 12 & 13 & 14 & 15 & 16 & 17 & 18 & 0 & 2\end{array}$

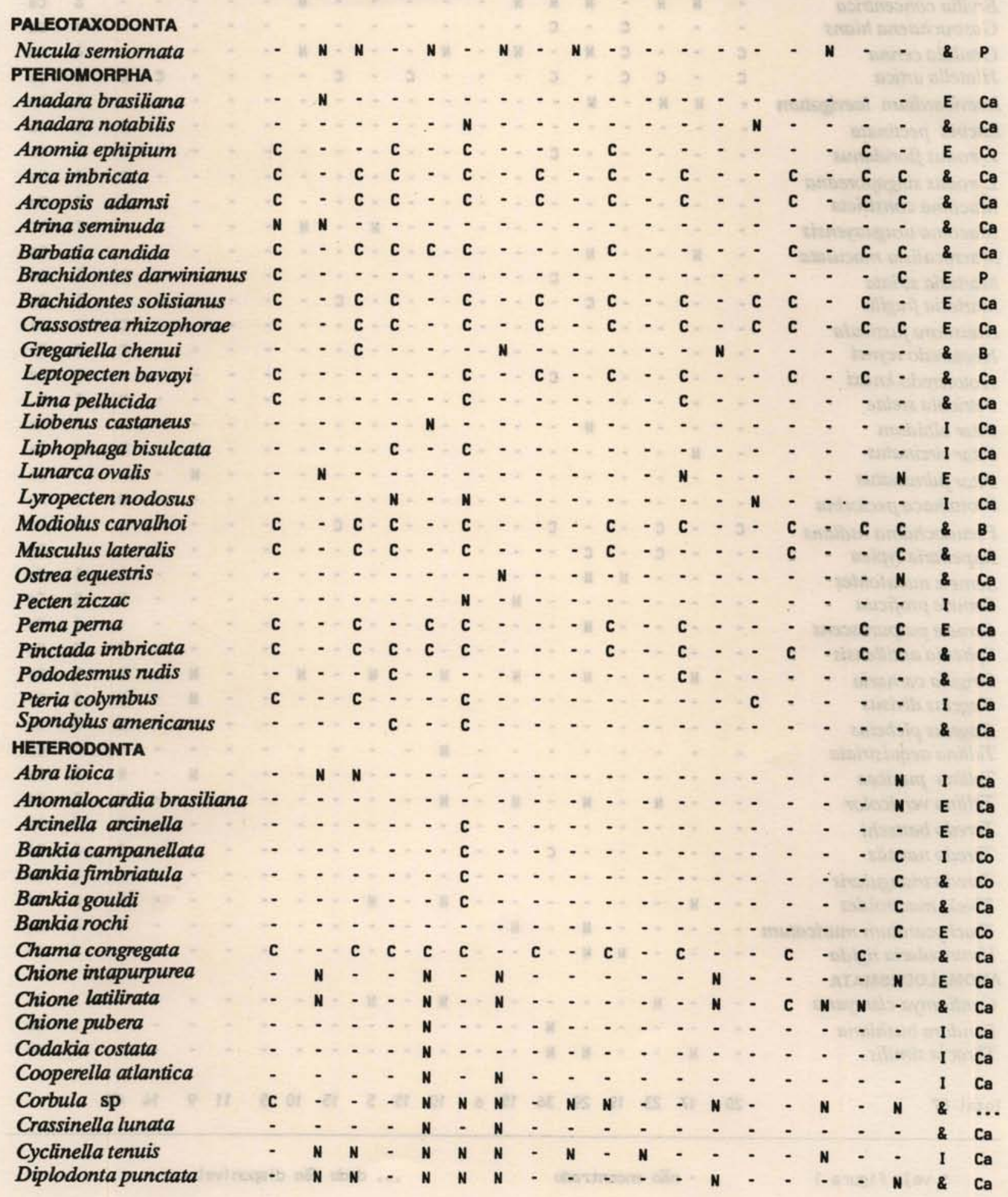


Tabela 2. Continuação

LOCAIS DE COLETA*

ESPECIES

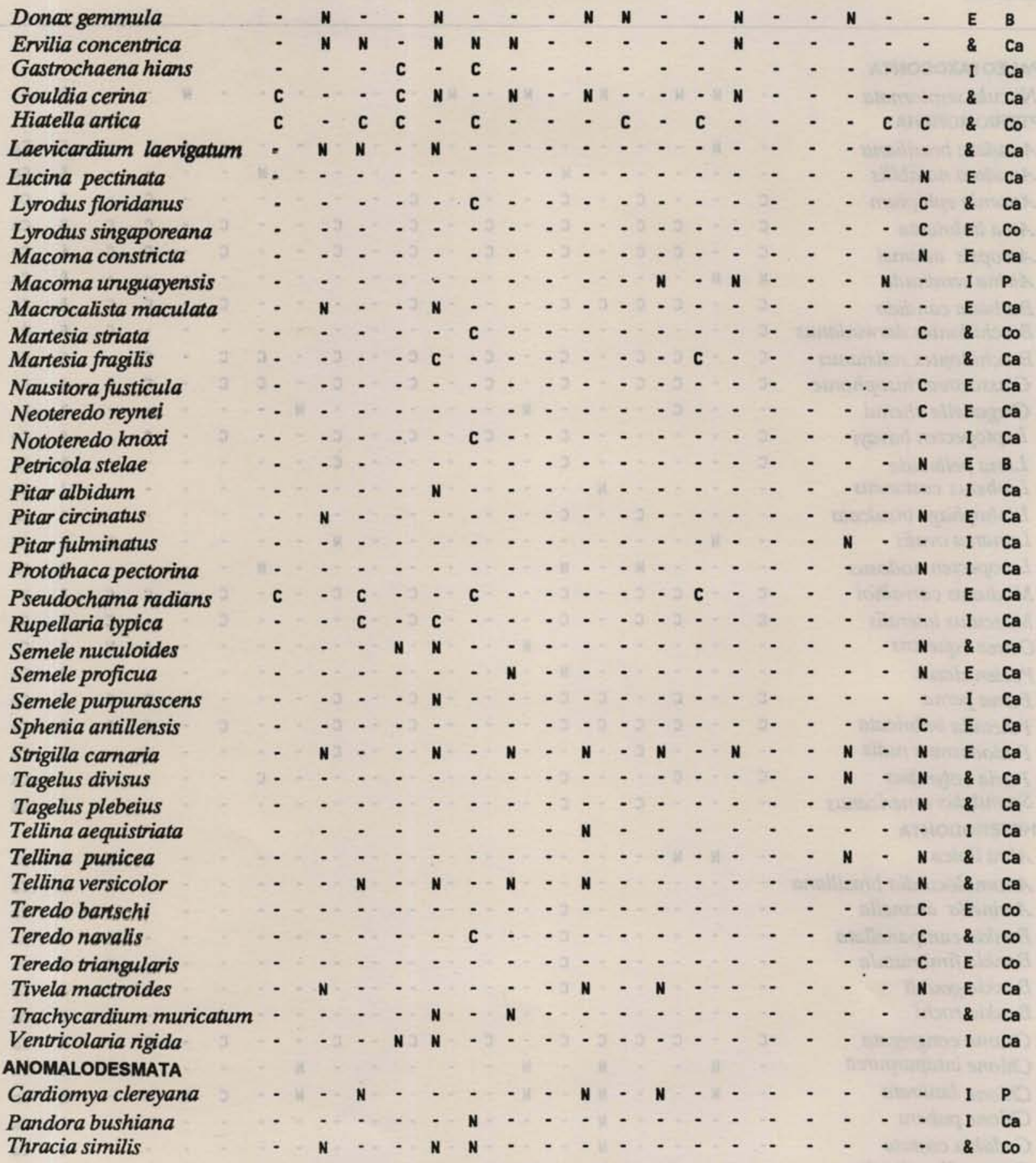

$\begin{array}{lllllllllllllllllll}\text { Total } 87 & 20 & 17 & 23 & 19 & 29 & 36 & 15 & 6 & 10 & 15 & 5 & 15 & 10 & 5 & 11 & 9 & 14 & 43\end{array}$ 
Lunarca ovalis foram frequientemente encontrados sobre a porção exposta de tubos do poliqueto Diopatra cuprea, na região entremarés.

Além das 195 espécies coletadas, são listadas na Tabela 6 outras 142 espécies registradas por vários autores para a regiāo (incluíndo a Ilha de São Sebastião e o Arquipélago de Alcatrazes) e não encontradas no presente trabalho.

\section{Discussão}

Em 1897, Thering listou 128 espécies de moluscos marinhos (48 gastropodes, 79 bivalves e 1 escaf6podo), entre animais vivos e conchas vazias, coletadas, sem referência aos locais específicos, num período de 3 a 4 meses no Canal de São Sebastiāo. Quarenta e duas destas espécies (6 gastrópodes e 36 bivalves) não foram reencontradas por nós. Devido à grande modificação na sistemática de Mollusca desde a publicação do trabalho deste autor, 12 espécies nominais não puderam ser correlacionadas com taxa modernos, sendo assinaladas na Tabela $6 \mathrm{com}$ um ponto de interrogação. É possível, portanto, que o número de espécies não reencontradas seja menor.

Luederwaldt \& Fonseca (1923) registraram para o Arquipélago de Alcatrazes 12 espécies de moluscos marinhos (8 gastrópodes, 3 bivalves e 1 poliplacóforo), dos quais somente nāo reencontramos uma espécie de gastrópode.

Luederwaldt (1929 a,b) apresenta 13 espécies da malacofauna local (9 gastrópodes, 3 bivalves e um escafópodo), incluindo quatro espécies de gastrópodes e duas de bivalves não reencontradas por nós.

Em um estudo da macrofauna de algumas praias da porçāo norte do Canal de São Sebastiāo, portanto fora da área de estudo do presente trabalho, Morgado et al. (1990) identificaram 13 espécies de moluscos (6 gastrópodes e 7 bivalves), 4 das quais (1 gastrópode e 3 bivalves) não estão incluídas na presente lista.

Tabela 3. Polyplacophora e Scaphopoda do Canal de São Sebastião, por local de coleta

SUBSTRATO - C: Consol idado; N: Não-consol idado

OCORRÊNCIA (O) - I: Infral itoral; E: Entremarés

DISTRIBUICÃ̃o ZOOGEOGRÁfICA (Z) - Ca: Caribeana; P: Patagônica; B: Endêmica da costa brasileirra

ESPECIES

LOCAIS DE COLETA*

$\begin{array}{llllllllllllllllllll}1 & 2 & 3 & 4 & 5 & 6 & 7 & 8 & 9 & 10 & 11 & 12 & 13 & 14 & 15 & 16 & 17 & 18 & 0 & Z\end{array}$

\section{POLYPLACOPHORA \\ Calloplax janeirensis Chaetopleura angulata Chaetopleura asperrima Ischnochiton striolatus}

Total 4

\section{SCAPHOPODA}

Dentalium disparile

Total 1

$$
\begin{aligned}
& \text { C - C }- \text { C }-- \text { C }- \text { C }--C-C-E \text { Ca }
\end{aligned}
$$

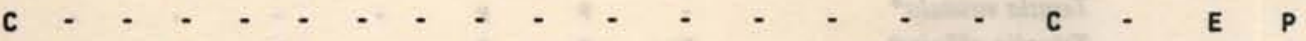

$$
\begin{aligned}
& \text { C - - C - C }- \text { C }- \text { C }- \text { C }- \text { - }- \text { - }- \text { C E B } \\
& \text { C }-\mathrm{C} C-\mathrm{C}-\mathrm{C}-\mathrm{C}-\mathrm{C}-\mathrm{C}-\mathrm{C}-\mathrm{C} \mathrm{C} \\
& \begin{array}{llllllllllllllllll}
4 & 0 & 1 & 3 & 0 & 3 & 0 & 2 & 0 & 3 & 0 & 3 & 0 & 0 & 2 & 0 & 3 & 2
\end{array}
\end{aligned}
$$


Tabela 4. Moluscos associados a algas, no Canal de São Sebastião

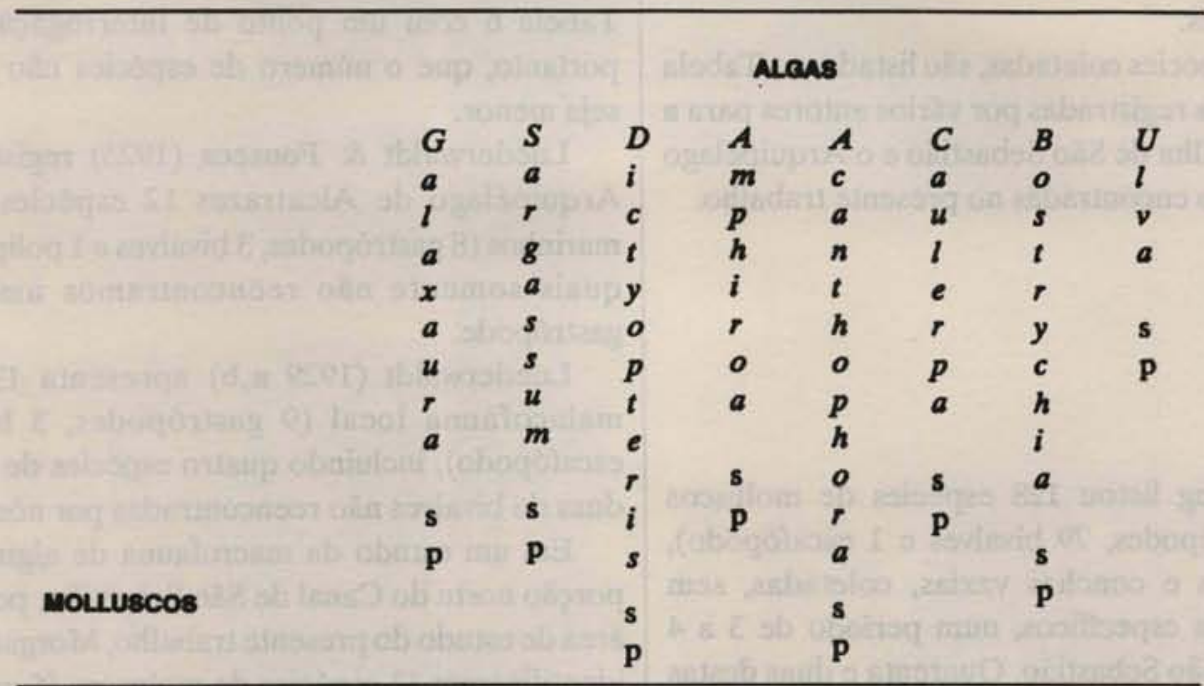

QASTROPODA

Anachis lynato Astraea phoebia*

Astyris lunata*"

Bittium varium*

Bulla striata*

Calliostoma jujubinum*

Calliostoma pulchrum*

Caecum pulchellum

Caecum yyssotitum

Cerithiopsis greeni *

Cerithium atratum*

Cerithium ebumeum *

Columbella mercatoria*

Costoanachis catenata\#

Costoanachis sertularianum

Engina turbinella*

Fissurella clenchi*

Leucozonia nassa*

Littorina flava*

Mitrella argus*

Odostomia seminuda

Parvanachis obesa

Seila adamsi

Tegula viridula*

Tricolia affinis\#

Turbonilla abrupta

BNALVIA

Chione latilinata*

Gregariella chenui

Hiatella antica

Leptopecten bavayi

Musculus lateralis

Modiolus canvalhoi

Pema perna*

Petricola stalae

Rupellaria typica

Sphenia antillensis

Total 36

17

15

10

10

15

3

2

2

* encontrecos exclueivemente no fital

- joven
P presente

- não encontrada 
Tabela 5. Moluscos associados a invertebrados, no Canal de São Sebastlão

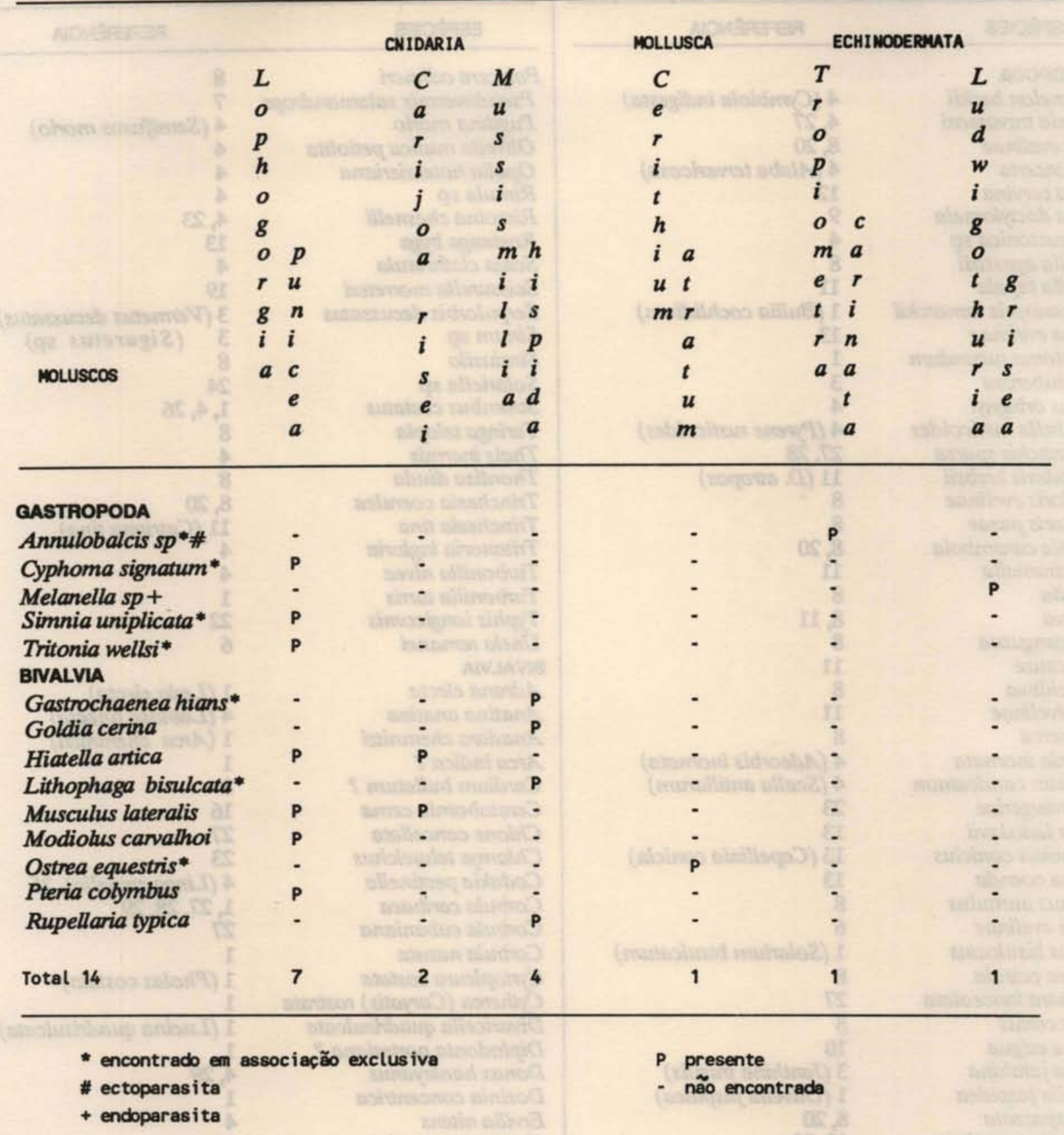

Amaral et al. (1990) encontraram, entre 1987 e 1989, 28 espécies de moluscos (12 gastrópodes e 16 bivalves) em algumas das praias estudadas no presente trabalho; destas, espécies sete (3 gastrópodes e 4 bivalves) não haviam sido coletadas por nós. Apesar disto, o número de espécies citado por estes autores é muito reduzido, quando comparado aos resultados por nós obtidos. Na regiāo entremarés, esses autores registraram apenas quatro espécies na Praia de Barequeçaba, 13 na Baía do Araçá, dois na Praia Grande, e nenhuma nas Praias do Segredo e Preta, enquanto encontramos, entre 1982 e 1983, um numero de $21,29,13,25$, e 10 espécies, respectivamente, em cada um dos locais acima citados. Montouchet (1988) considerou a ausência de Neritina virginea, outrora abundante na Baía do Araçá (mas já encontrada em baixa freqüência no presente trabalho), a alta freqüência de conchas vazias e "a fragilidade anormal das conchas de bivalves" decorrentes da ação de poluentes ou de outras perturbaçōes ambientais. Após as drásticas interferências antrópicas de 1988 e 1989 na Baía do 
Tabela 6. Espécies citadas para a região de São Sebastiăo (incluindo a llha de São Sebastião e o Arquipélago de Alcatrazes) e năo encontradas no presente trabalho

\begin{tabular}{|c|c|}
\hline ESPÉEIES & REFERENCIA \\
\hline GASTROPODA & \\
\hline Adelomelon beckii & 4 (Cymbiola indigesta) \\
\hline $\begin{array}{l}\text { Agaronia travassosi } \\
\text { Aglaja evelinae }\end{array}$ & $\begin{array}{l}4,27 \\
8,20\end{array}$ \\
\hline Alaba incerta & 4 (Alaba tervaricosa) \\
\hline Aplysia cervina & 12 \\
\hline Aplysia dactylomela & is \\
\hline Architeactonica sp & 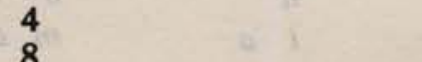 \\
\hline Berthella agassizii & 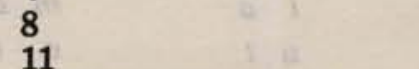 \\
\hline Berthella tupala & 11 \\
\hline Buccinanopsis lamarckii & 1 (Bullia cochlidium) \\
\hline Cadlina evelinae & 13 \\
\hline Calliostoma jucundum & 1 \\
\hline $\begin{array}{l}\text { Cassis tuberosa } \\
\text { Circulus orbignyi }\end{array}$ & $\begin{array}{l}3 \\
4\end{array}$ \\
\hline Columbella nusticoides & 4 (Pyrene rusticoides) \\
\hline Costoanachis sparsa & 27,28 \\
\hline Dendrodoris krebsii & 11 (D. atropos) \\
\hline Discodoris evelinae & 8 \\
\hline Discodoris pusae & 8 \\
\hline Doridella carambola & 8,20 \\
\hline Doto caramella & 11 \\
\hline Doto pita & 8 \\
\hline Doto uva & $\begin{array}{l}8,11 \\
8\end{array}$ \\
\hline Elysia canguzua & \\
\hline $\begin{array}{l}\text { Elysia caure } \\
\text { Elysia chitwa }\end{array}$ & $\begin{array}{l}11 \\
8\end{array}$ \\
\hline $\begin{array}{l}\text { Elysia chitwa } \\
\text { Elysia evelinae }\end{array}$ & 11 \\
\hline Elysia serca & \\
\hline Episcynia inornata & 4 (Adeorbis inornata) \\
\hline Epitonium candeanum & 4 (Scalla antillanum) \\
\hline Erato maugeriae & 23 \\
\hline Etidoris ladislavii & 13 (13 Canellinia conicla) \\
\hline Eubranchus coniclus & 13 (Capellinia conicla) \\
\hline Facelina coenda & 13 \\
\hline Favorinus auritulus & 8 \\
\hline Ganitus evelinae & \\
\hline Heliacus bisulcatus & 1 (Solarium bisulcatum) \\
\hline Hermaea coirala & 8 \\
\hline Ithycythara lanceolata & 27 \\
\hline Janolus comis & 8 \\
\hline Janthina exigua & \\
\hline $\begin{array}{l}\text { Janthina janthina } \\
\text { Jaspidella jaspidea }\end{array}$ & 3 (Janthina fragilis) \\
\hline $\begin{array}{l}\text { Jaspidella jaspidea } \\
\text { Joruna spazzola }\end{array}$ & $\begin{array}{l}1 \text { (Olivella jaspidea) } \\
8,20\end{array}$ \\
\hline Lamellaria perspicua & $\begin{array}{l}8,20 \\
10,23\end{array}$ \\
\hline Litiopa melanostoma & 4 \\
\hline Lophodoris scala & 17 \\
\hline Lomanotus phiops & 11 \\
\hline Lucapina sowerbii & 2 (Fissurella cancellata), 4, 5 \\
\hline $\begin{array}{l}\text { Lucapinella limatula } \\
\text { Mysouffa cumingii }\end{array}$ & $\begin{array}{l}4 \\
18 \text { (Tomlinula cumingii), } 20\end{array}$ \\
\hline $\begin{array}{l}\text { Mysouffa cumingii } \\
\text { Okenia implexa }\end{array}$ & \\
\hline Paraseptaria panqueca & 10 \\
\hline Peltodoris greeleyi & 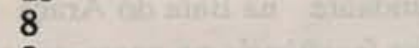 \\
\hline Phyllaplysia engeli & 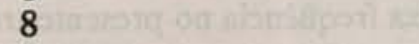 \\
\hline Piseinotecus divae & 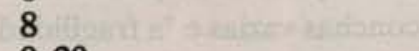 \\
\hline $\begin{array}{l}\text { Pleurobranchaea hedgpethi } \\
\text { Phuscula quica }\end{array}$ & atiormentis \\
\hline $\begin{array}{l}\text { Phuscula quica } \\
\text { Polycera aurisula }\end{array}$ & 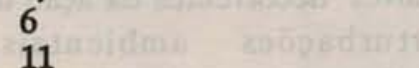 \\
\hline Polycera aurisula & 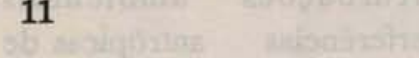 \\
\hline
\end{tabular}

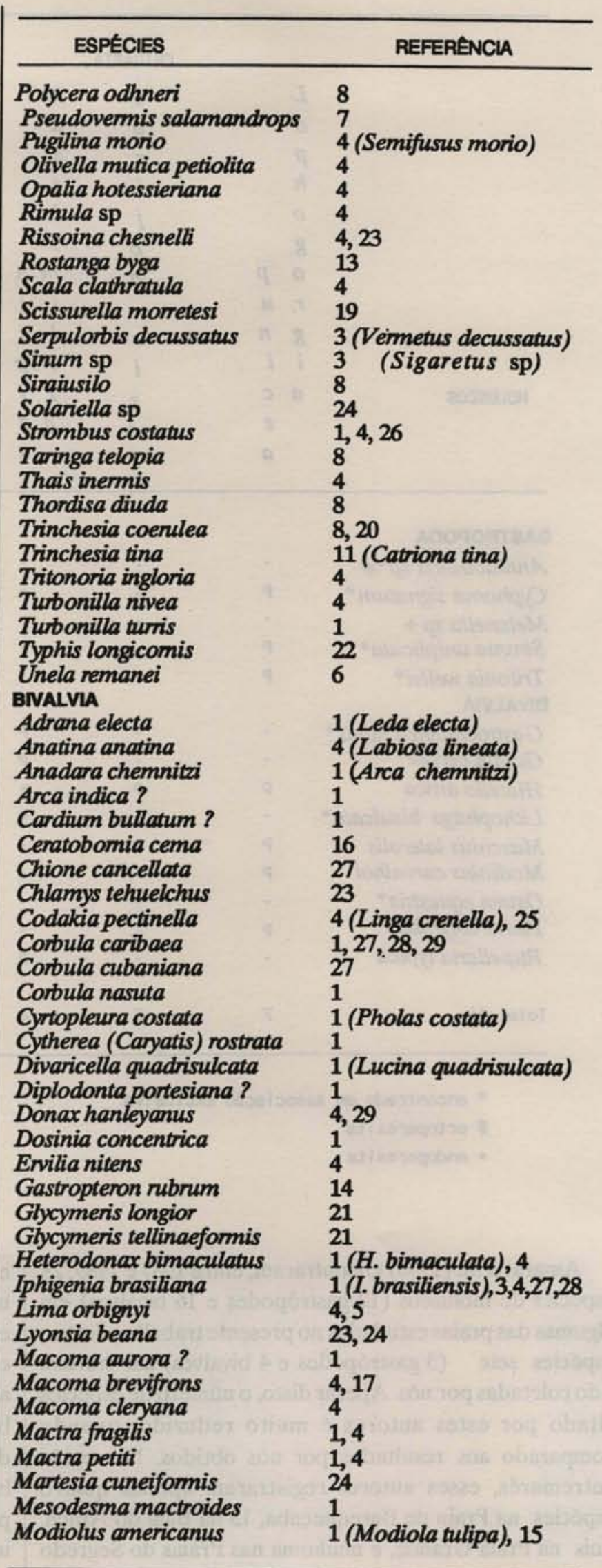


Tabela 6. Continuação

\begin{tabular}{ll}
\hline \multicolumn{1}{c}{ ESPECIES } & \multicolumn{1}{c}{ REFERENCIA } \\
\hline Musculus viator & 1 (Modiolaria viator), 15 \\
Mytella falcata & 1 (Modiola falcata), 15 \\
Mytella guyanensis & 1 (Modiola brasiliensis),15 \\
Noetia bisulcata & 1 (Arca martini) \\
Pandora derbyi ? & 3 \\
Papyridae soleniformis & 4 (Papyridae spinoza) \\
Phlyctiderma semiaspera & 1 (Diplodonta semireticulata) \\
Pitar rostratus & 4 \\
Plicatula gibbosa & 1 (Plicatula ramosa) \\
Sanguinolaria cruenta & 17 \\
Sanguinolaria sanguinolenta & 1,29 \\
Semele reticulata? & 1 \\
Semele variegata ? & 1 \\
Solecurtus comingianus & 4 \\
Solecurtus sanctamantae & 4 \\
Solen rostriformis & 4 \\
Solen tehuelchus & 27,28 \\
Strigilla pisiformis & 1 \\
Tellina angulosa & 1 (Tellina striata) \\
Tellina lineata & 1 \\
Tellina martinicensis & 1 (Gouldia martinicensis) \\
Tellina radiata & 1 (Tellina brasiliana) \\
Tellina sandix & 4 (Angulus exilis) \\
Venus cribaria? & 1 \\
Venus dysera? & 1 \\
Venus portesiana ? & 1 \\
Venus nugosa? & 1 \\
sCAPHOPODA & 1 \\
Dentalium americanum & 4 (D.texasianum rioense) \\
& \\
\hline &
\end{tabular}

1 Ihering (1897)

16 Narchi (1966)

2 Luederwaldt \& Fonseca (1923) 17 Ditadi (1969)

3 Luederwaldt $(1929 \mathrm{a}, \mathrm{b})$

4 Morretes (1949)

5 Morretes (1953)

6 Marcus, E (1953)

7 Marcus, E. B. R. (1953)

8 Marcus, E. (1955)

9 Marcus \& Marcus (1955)

10 Marcus, E. B. R. (1956)

11 Marcus, E. (1957)

12 Marcus \& Marcus (1957)

13 Marcus, E. (1958)

14 Marcus \& Marcus (1960)

15 Klappenbach (1965)

18 Marcus, E. B. R. (1972)

19 Montouchet (1972)

20 Marcus, E. B. R (1977)

21 Penna-Neme (1978)

22 Penna-Neme \& Leme (1978)

23 Duarte (1980)

24 Morgado (1980)

25 Penna-Neme \& Cruz-Natali (1984)

26 Domaneschi (1986)

27 Amaral et al (1990)

28 Morgado et al. (1990)

29 Shimizu (1991)

Observaçōes:

- o nome entre parênteses após uma referéncia é aquele sob o qual a espécie foi originalmente registrada.

- o ponto de interrogaçăo após o nome da espécie indica dúvida quanto ao status atual do taxon.

Araçá, observa-se uma lenta recuperaçāo do local e, após mais de dois anos, muitas espécies estão em densidade muito baixa ou mesmo ausentes (Migotto \& Tiago, obs. pessoal). A poluiçāo foi também, sugerida como um agente redutor do número de espécies e da abundância de indivíduos por Amaral et al. (1987) para a fauna de anelídeos poliquetos da região entremarés da Praia do Flamengo, Ubatuba (SP).
Por outro lado, os dados de Shimizu (1991) para a Praia de Barequeçaba, obtidos entre 1987 e 1989, não diferem tanto dos nossos quanto os de Amaral et al. (1990). Shimizu (op. cit.) coletou cinco espécies de gastrópodes e 12 de bivalves, das quais não encontramos três de gastrópodes e quatro de bivalves.

Parte das diferenças no número de espécies citadas por estes autores pode decorrer também do emprego de metologias de coleta diversas.

\section{Conclusões}

Ainda que existam diferenças metodológicas, a comparação dos dados do presente trabalho, obtidos no período de 1982 a 1983, com os de Amaral et al.(1990) e de Shimizu (1991), obtidos no período de 1987 a 1989, em relação à regiāo entremarés de cinco praias arenosas estudadas nesses dois períodos, mostra uma redução no número de espécies de moluscos. A interferência ambiental crônica no Canal de São Sebastiāo é, aparentemente, um dos fatores responsáveis pela diminuição desta diversidade.

\section{Agradecimentos}

Aos Drs Lícia Penna-Neme (in memoriam) e José Luiz Moreira Leme pelo auxilio indispensável na identificação dos moluscos e na consulta à coleção do Museu de Zoologia da USP, à Dra Eveline Marcus (in memoriam) (Departamento de Zoologia, USP) pela identificação dos opistobrânquios, ao Dr Anders Waren (Naturhistoriska Riksmuseet, Stockholm, Suécia) pela identificação dos eulimídeos, ao Dr Piet Kaas (Nationaal Natuurhistorisch Museum, Leiden, Holanda) pela identificação dos poliplacoforos, e ao Dr Osmar Domaneschi (Departamento de Zoologia, USP) pelas valiosas sugestōes.

\section{Referências bibliográficas}

ABBOTT, R. T. 1974. American seashells. $2^{\text {nd }}$ ed. New York, Van Nostrand Reinhold. 663p.

AMARAL, A. C. Z.; NONATO, E. F. \& MORGADO, E. H. 1987. Alteraçōes na fauna de anelídeos poliquetos da Praia do Saco da Ribeira, Ubatuba-SP. In: SIMPÓSIO DE ECOSSISTEMAS DA COSTA SUL ESUDESTE BRASILEIRA, Cananéia, 1987. São Paulo, Academia de Ciências do Estado de São Paulo. v.3, p.244-257. 
AMARAL, A. C. Z. ; MORGADO, E. H.; LOPES, P. P.; BELÚCIO, L. F.; LEITE, F. P. P. \& FERREIRA, C. P. 1990. Composition and distribution of the intertidal macrofauna of sandy beaches on São Paulo coast. In: SIMPÓSIO DE ECOSSISTEMAS DA COSTA SUL E SUDESTE BRASILEIRA, 2., Águas de Lindóia, 1990. São Paulo, Academia de Ciências do Estado de São Paulo. v. 3, p.258-279.

API-AMERICAN PETROLEUM INSTITUTE. 1985. Oil spill response: options for minimizing adverse ecological impacts. Washington, D.C., American Petroleum Institute. 98p.

CEBIMAR. 1991. Dados ambientais - 1979/1991. São Sebastiāo, Centro de Biologia Marinha-USP. 22p.

CETESB-GOE/DAEM 1989. Relatório de acidentes São Sebastiāo. Listagem 09/01/78 - 23/10/1989. São Paulo, Companhia de Tecnologia de Saneamento Ambiental. 2p.

DITADI, A. S. F. 1969 . Contribuição ao estudo da ecologia e fisiologia de Lissomyema exilii (F. Müller, 1883) (Echiura). Tese de doutorado. Universidade de Sāo Paulo, Faculdade de Medicina. 128p.

DOMANESCHI, O. 1986. Reencontro de Strombus costatus Gmelin, 1791 (Gastropoda, Strombidae) no litoral do Estado de São Paulo, Brasil. Bolm Zool, Univ. S Paulo, 10:311-316.

DUARTE, L. F. L. 1980. A endofauna da esponja Zygomycale parishii (Bowerbank) (composiçāo, dominância, diversidade e natureza da associação). Dissertação de mestrado. Universidade Estadual de Campinas, Instituto de Biologia. 103p.

FURTADO, V. V. 1978. Contribuiçāo ao estudo da sedimentação atual no Canal de São Sebastiāo, Estado de São Paulo. Tese de doutorado. Universidade de São Paulo, Instituto de Geociências. 2 v.

IHERING, H. von 1897. A Ilha de São Sebastião. Revta Mus. paul., 2:129-171.

KLAPPENBACH, M. A. 1964. Nueva Olivella (Moll. Gastr.) de la costa brasileña del Estado de San Pablo. Comun. zool. Mus. Hist. nat. Montev., $8(101): 1-5$.

1965. Lista preliminar de los Mytilidae brasileños con claves para su determinación y notas sobre su distribuición. Anais Acad. brasil. Ciênc., 37(Supl.):325-352.
KLAPPENBACH, M. A. 1966. Nueva especie de Modiolus (Mollusca, Pelecypoda) de la costa brasileña. Papéis Dep. Zool., S Paulo, 19(21):251-257.

LUEDERWALDT, H. 1929a. Resultados de uma excursão scientífica à Illha de São Sebastiāo no littoral do Estado de São Paulo e em 1925. Revta Mus. paul., 16:1-79.

1929b. Errata, addiçōes e modificaçōes aos "Resultados de uma excursão scientífica à Ilha de São Sebastiāo em 1925". Revta Mus. paul., 16:1111-1119.

\& FONSECA, J. P. 1923. A Ilha dos Alcatrazes. Revta Mus. paul., 13:439-513.

MARCUS, E. 1953. Three Brazilian sand-opisthobranchia. Bolm Fac. Fil. Ciênc. Letr., Zool., (18):165-203.

1955. Opisthobranchia from Brazil. Bolm Fac. Fil. Ciênc. Letr., Zool., (20):89-261.

1957. On Opistobranchia from Brazil (2). J. Linn. Soc., Zool., 13:390-486.

1958. On Western Atlantic opistobranchiate gastropods. Am. Mus. Novit., 1906:1-82.

\& MARCUS, E. 1955. Sea-hares and side-gilled slugs from Brazil. Bolm Inst. oceanogr., S Paulo, 6(1-2):3-49.

\& MARCUS, E. 1957. Notes on Aplysia. Bolm Inst. oceanogr., S Paulo, 8(1-2):3-22.

\& MARCUS, E. 1960. Opistobranchs from American Atlantic warm waters. Bul. mar. sci. Gulf Caribb., 10(2):130-203.

MARCUS, E. B. R. 1953. The opisthobranch Pseudovermis from Brazil. Bolm Fac. Fil. Ciênc. Letr., Zool., (18):109-127.

1956. On some Prosobranchia from the coast of São Paulo. Bolm Inst. oceanogr., S Paulo, 7(1-2):3-29.

1972. On some Acteonidae (Gastropoda, Opisthobranchia). Papéis Zool., S Paulo, 25(19):167-188.

1977. An annotated checklist of the Western Atlantic warm water opisthobranchs. J. Moll. Stud., Supl. 4:1-22. 
MONTOUCHET, P. C. 1972. Three new species of Scissurellidae (Gastropoda, Prosobranchia) from the coast of Brazil. Bolm Inst. oceanogr., S Paulo, 21:1-13.

1988. A fauna de molucos em sedimentos do Araçá na zona das marés (São Sebastiāo, SP). In: MINI-SIMPÓSIO DE BIOLOGIA MARINHA, 7., Sāo Sebastiāo, 1988. Programa/Resumo. São Sebastiāo, Centro de Biologia Marinha da Universidade de São Paulo. p. 29.

MORGADO, E. H. 1980. A endofauna de Schizoporella unicomis (Johnston, 1847) (Bryozoa), no litoral norte do Estado de São Paulo. Dissertação de mestrado. Universidade Estadual de Campinas, Instituto de Biologia. 126p.

; AMARAL, A. C. Z; BELÚCIO, L. F.; LOPES, P. P.; FERREIRA, C. P. \& LEITE, F. P. P. 1990. The intertidal macrofauna of Sāo Francisco complex beaches (São Sebastiāo, SP). In: SIMPÓSIO DE ECOSSISTEMAS DA COSTA SUL E SUDESTE BRASILEIRA, 2., Águas de Lindóia, 1990. São Paulo, Academia de Ciências do Estado de São Paulo. v.3, p.314-325.

MORRETES, F. L. 1949. Ensaio de catálogo de moluscos do Brasil. Archos Mus. parana., 7:5-216.

1953. Adenda e corrigenda ao ensaio de catálogo dos molusculos do Brasil. Archos Mus. parana., 10:37-76.

NARCHI, W. 1966. The functional morphology of Ceratobomia cema, new species of the Erycinacea (Mollusca, Eulamellibranchiata). Anais Acad. brasil. Ciênc., 38(3/4):513- 524.

PALACIO, F. L. 1982. Revisión zoogeográfica marina del sur del Brasil. Bolm Inst. oceanogr., S Paulo, 31:69-92.

PENNA, L. 1971. Novas espécies e registros de Limidae e Carditidae (Pelecypoda) no litoral brasileiro. Papéis Zool., S Paulo, 24(11):155-159.

PENNA-NEME, L. 1978. Os Glycymerididae da costa brasileira (Mollusca, Bivalvia). Papéis Zool, S Paulo, 32(5):59-70.
PENNA-NEME, L. \& LEME, J. L. M. 1978. Novas espécies e novas ocorrências de gastrópodos marinhos na costa brasileira (Prosobranchia, Neogastropoda). Papéis Zool., S Paulo, 31(18):283-297.

PENNA-NEME, L. \& CRUZ-NATALI, M. L. 1984. Phacoides (Parvilucina) coupoisi Klein, 1967, novo sinônimo de Codakia (Ctena) pectinella (C. B. Adams, 1852), e algumas consideraçōes sobre os Lucinidae brasileiros (Mollusca, Bivalvia). Papéis Zool., S Paulo, 35(14):153-158.

RIGHI, G. 1967. Sôbre Polyplacophora do litoral brasileiro. Papéis Zool., S Paulo, 20(9):85-98.

RIOS, E. C. 1985. Seashells of Brazil: Rio Grande, Fundação Cidade do Rio Grande/ Fundação Universidade do Rio Grande/ Museu Oceanografico. 328 p.

SCHAEFFER-NOVELLI, Y. 1990. Vulnerabilidade do litoral norte do Estado de Sāo Paulo a vazamentos de petróleo e derivados. In: SIMPÓSIO DE ECOSSISTEMAS DA COSTA SUL E SUDESTE BRASILEIRA, 2., Águas de Lindóia, 1990. São Paulo, Academia de Ciências do Estado de São Paulo. v.2, p.375-399.

SHIMIZU, R. M. 1991. A comunidade de macroinvertebrados da regiāo entre marés da Praia de Barequeçaba, Sāo Sebastiāo, SP. Dissertação de mestrado. Universidade de São Paulo, Instituto de Biociências. 72p.

TIAGO, C. G. 1989. Sobre uma comunidade de teredinídeos (Mollusca, Bivalvia) em São Sebastiāo (SP), Brasil. Dissertaçāo de mestrado. Universidade de São Paulo, Instituto de Biociências. 88p.

(Manuscrito recebido 6 julho 1992; revisto 15 março de 1993; aceito 13 agosto 1993) 